\title{
Erratum to: Strength training stops bone loss and builds muscle in postmenopausal breast cancer survivors: a randomized, controlled trial
}

\author{
Kerri M. Winters-Stone $\cdot$ Jessica Dobek • \\ Lillian Nail • Jill A. Bennett • Michael C. Leo • \\ Arpana Naik • Anna Schwartz
}

Published online: 19 April 2011

(C) Springer Science+Business Media, LLC. 2011

\section{Erratum to: Breast Cancer Res Treat DOI 10.1007/s10549-011-1444-z}

Regretfully, we have identified a typographical and a computational error in the results displayed for the effect modification of adjuvant hormone therapy on lean body mass reported in our manuscript, "Strength training stops bone loss and builds muscle in postmenopausal breast cancer survivors: a randomized, controlled trial." The corrected data are below:

Coefficient of group by AI vector on slope of time $=$ $-955.55, \mathrm{SE}=367.15, t(96)=-2.60, P=0.01$.

We originally reported an exercise group by hormone treatment interaction such that lean mass increased in the POWIR group more among women treated with aromatase

The online version of the original article can be found under doi:10.1007/s10549-011-1444-z.

K. M. Winters-Stone $(\bowtie) \cdot J$. Dobek $\cdot$ L. Nail

J. A. Bennett · M. C. Leo

School of Nursing, Oregon Health \& Science University,

3455 SW US Veteran's Hospital Rd., Mailcode: SN-ORD,

Portland, OR 97239, USA

e-mail: wintersk@ohsu.edu

\section{A. Naik}

School of Medicine, Oregon Health \& Science University,

Portland, OR, USA

\section{A. Schwartz}

School of Nursing, University of Washington, Seattle, WA, USA inhibitors (AIs) than those who were not treated with AIs, while lean mass did not change among FLEX group participants regardless of AI use. As shown in the corrected Fig. 3, lean body mass increased in POWIR participants who were not receiving hormone treatment and FLEX participants receiving AIs but did not change over time in POWIR participants treated with AIs and women in the FLEX group who were not receiving hormone treatment.

Contrary to our original conclusion that resistance training and AIs may act synergistically to increase lean body mass, our results are not consistent with the existing literature and suggest the possibility that AIs may not have the same effects on lean body mass across different forms of exercise.

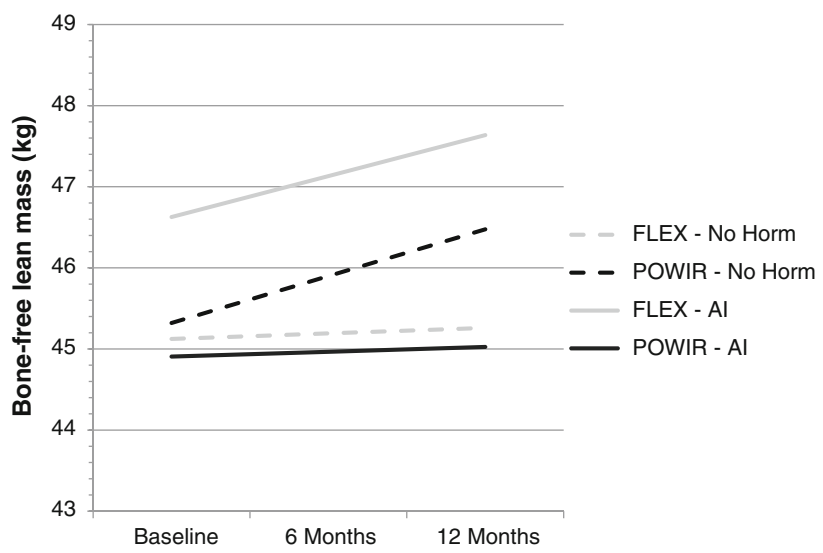

Fig. 3 Changes in bone-free lean mass over 12 months by exercise group between current users of $\mathrm{AI}$ and non-hormone users using predicted means from HLM analyses 